Bipolar Spectrum Psychopathology is Associated with Altered Emotion Dynamics Across Multiple Timescales

Sarah H. Sperry ${ }^{1} \quad$ Thomas R. Kwapil ${ }^{1,2}$

${ }^{1}$ University of Illinois at Urbana-Champaign

${ }^{2}$ University of North Carolina at Greensboro

In press, Emotion.

This is an unedited manuscript accepted for publication. The manuscript will undergo copyediting, typesetting, and review of resulting proof before it is published in its final form.

Author Note

Correspondence concerning this article should be addressed to Sarah Sperry, Department of Psychology, University of Illinois at Urbana-Champaign, Champaign, IL, 61820. Contact: ssperry2@illinois.edu.

We would like to acknowledge Haley E. Clark and Elizabeth E. Spurlock for aiding in data collection, cleaning, and organization.

Abstract 
Emotion dysregulation is a core feature of bipolar spectrum psychopathology and may confer risk for poor outcomes or progression along the bipolar spectrum. However, previous research on bipolar psychopathology has primarily concentrated on characterizing distinct mood episodes and failed to characterize micro-level dynamics of the experience of emotion. This is the first study to our knowledge to comprehensively examine the extent to which bipolar spectrum psychopathology, as measured by the Hypomanic Personality Scale (HPS), is associated with altered dynamics of positive (PA) and negative affect (NA) across multiple timescales. Young adults $(n=233)$ oversampled for high HPS scores $(>1.5 \mathrm{SD}$ ) completed self-report questionnaires and 14 days of experience sampling questionnaires assessing high and low arousal NA and PA. Four emotion dynamics (reactivity, variability, instability, inertia) were computed from each participant's time-series. As predicted, HPS were positively associated with variability and instability of high arousal NA and PA both within- and between-days (over-and-above meanlevels of emotions, depression, and neuroticism). Further, HPS scores were associated with large fluctuations in low but not high arousal NA and moderated stress-reactivity. Specifically, those high scorers on the HPS were more likely to report feeling like their emotions were out of control (but not high intensity NA) when experiencing stress. Contrary to expectation, HPS scores were unassociated with inertia of high arousal PA. Findings indicated that micro-level emotion dynamics are disrupted across multiple timescales in those high in bipolar spectrum psychopathology. Examining emotion dynamics should enhance understanding of risk for bipolar disorders and facilitate development of mood-monitoring interventions. Keywords: Bipolar, Emotion, Affect, Dynamics, Experience Sampling Methodology, Time Series Analysis 


\section{Bipolar Spectrum Psychopathology is Associated with Altered Emotion Dynamics Across Multiple Timescales}

Bipolar disorders, categorically defined, affect approximately $4 \%$ of the population (Merikangas et al., 2007), are associated with severe impairment and premature mortality (Rshoanaei-Moghaddam \& Katon, 2009), and account for more than 150 billion dollars in direct and indirect costs in the US annually (Dilsaver, 2011). However, it is estimated that up to $9 \%$ of the population experiences subthreshold bipolar symptoms (Angst, Gamma, Benazzai, et al., 2003) that are associated with poor functioning (Judd \& Akiskal, 2003) and increased risk of bipolar disorders (Kwapil et al., 2000; Walsh, DeGeorge, Barrantes-Vidal, \& Kwapil, 2015). Furthermore, taxonomic analyses suggest that bipolar disorders are better represented by a spectrum of symptoms and impairment than discrete categorical diagnoses (Ahmed, Green, Clark, Stahl, \& McFarland, 2011; Prisciandaro \& Tolliver, 2015). Thus, current models increasingly conceptualize bipolar psychopathology as a spectrum, ranging from subclinical expression to full-blown mania.

Emotion dysregulation is a core feature of bipolar disorders as evidenced by the presence of hypomanic, manic, and/or depressive episodes. During depressive episodes, people typically experience heightened negative affect (NA), whereas during hypomania or mania, people display elevated positive affect (PA) and/or irritability (American Psychiatric Association, 2013). Based on this description, emotion dysregulation has typically been conceptualized as episodic disturbances in mood. Euthymia, in contrast, represents the absence of elevated mood. However, lab-based studies show that bipolar spectrum disorders are associated with affective hyperreactivity (Alloy, Abramson, Walshaw, Keyser, \& Gerstein, 2006; M'Bailara et al., 2009), poor affective recovery (Farmer et al., 2006), and heightened lability (Angst, Gamma, \& Endrass, 
2003; Hofmann \& Meyer, 2006; Lovejoy \& Steuerwald, 1995) even during euthymia.

Furthermore, subthreshold bipolar spectrum psychopathology, in non-clinically ascertained samples, is associated with elevated levels of energetic-enthusiasm, dysphoria, and irritability in daily life (Kwapil et al., 2011; Sperry \& Kwapil, 2017; Walsh, Royal, Brown, Barrantes-Vidal, \& Kwapil, 2012). Those high on bipolar spectrum psychopathology also report that their emotions feel out of control and their mood fluctuates "up and down" (Kwapil et al., 2011; Sperry \& Kwapil, 2017), especially in stressful or unpleasant situations (Sperry \& Kwapil, 2019). Taken together, this suggests that outside the context of clinical mood episodes, bipolar spectrum psychopathology is associated with affective lability.

Affective lability is linked to numerous negative outcomes. Angst, Gamma, and Endrass (2003) found that "mood ups and downs" were a risk factor for the development of bipolar disorders. Notably, inter-episodic affective lability predicted worse functioning and more severe symptoms in those who had already developed bipolar spectrum disorders (Henry et al., 2001, 2008). Lastly, individuals with bipolar disorders who are characterized by a "mixed" profile with more temporally unstable mood had higher rates of psychosis, substance use, and rapid cycling (Prisciandaro, Tolliver, \& DeSantis, 2018). Given that affective lability may predict the development of bipolar spectrum disorders and is associated with more severe psychopathology, understanding the nature and course of emotion dynamics in bipolar spectrum psychopathology is essential.

Real-time assessment of emotion dynamics in daily life offers a comprehensive method for examining affective lability. The measurement of emotion dynamics refers to examining time-dependent fluctuations in emotion in daily life (Kuppens, Allen, \& Sheeber, 2010). Affective reactivity, variability, instability, and inertia are emotion dynamics widely measured to 
characterize emotion dysregulation and affective lability in mood psychopathology. Affective reactivity, as measured by ESM, captures the extent to which individuals are more likely to experience a change in emotion in reaction to an increase in a proximal risk factor (e.g., stress; Stange, Kleiman, Mermelstein, \& Trull, 2019). Affective variability captures how much an individual's affect in daily life deviates in intensity from their own mean levels of affect (Eid \& Diener, 1999; Jahng, Wood, \& Trull, 2008). The extent to which individuals deviate away from their "core affect" is an important individual difference that has been widely linked to well-being and psychopathology over-and-above mean levels of emotions (Houben, Van Den Noortgate, \& Kuppens, 2015; Kuppens, Oravecz, \& Tuerlinckx, 2010). Affective instability captures whether an individual's affect fluctuates from moment-to-moment, and whether those fluctuations are large (Jahng et al., 2008). Although instability takes into account variability, it assesses the temporal dependency and frequency of fluctuations in affect. Lastly, affective inertia reflects the extent to which affect is a) resist to change, b) persistent over time, or c) fails to down-regulate following perturbation (Koval et al., 2015; Kuppens, Allen, et al., 2010).

The literature on emotion dynamics in bipolar spectrum psychopathology is limited and has primarily examined long-term changes in symptoms of depression and mania rather than moment-to-moment emotion dynamics. For example, studies examining the statistical patterns of depressive and manic symptoms over time reported that bipolar I disorder is characterized by highly non-linear time-series (Bonsall, Wallace-Hadrill, Geddes, Goodwin, \& Holmes, 2012; Cochran, McInnis, \& Forger, 2016; Steinacher \& Wright, 2013). Weekly depression scores across 220 weeks were highly variable and could not be predicted by the prior week's symptoms (Bonsall et al., 2012). Similarly, cluster analysis of patients with bipolar I and II disorders revealed "high mood instability" and "low mood instability" groups (Szmulewicz, Martino, \& 
Strejilevich, 2019). The high mood instability group had significantly more shifts between depression, euthymia, and hypomania/mania than the low instability group.

In a seven-day experience sampling methodology (ESM) study of non-clinical young adults, Sperry and Kwapil (2019) found that bipolar spectrum psychopathology, as measured by the Hypomanic Personality Scale (HPS; Eckblad \& Chapman, 1986), was associated with affective hyper-reactivity of NA, variability and instability of NA and PA, and was unassociated with affective inertia over-and-above mean levels of NA and PA. Although this was the first study to examine short-term dynamics in bipolar spectrum psychopathology, it was preliminary in that, 1) all analyses were exploratory, 2) participants were not over-sampled for scores on the HPS, and 3) the ESM protocol did not adequately assess pleasant experiences/events in daily life. Sperry, Walsh, and Kwapil (2019) then examined whether patterns of emotion dynamics concurrently and prospectively predicted HPS scores, hypomanic episodes, and bipolar spectrum diagnoses three-years later. They replicated findings from Sperry and Kwapil (2019) in that HPS scores were associated with variability and instability of NA and PA over-and-above mean levels at baseline. Furthermore, instability of NA and PA predicted symptoms and diagnoses of bipolar spectrum disorders three years later. However, this study did not assess high and low arousal NA and PA and had a limited set of ESM items to assess affective reactivity.

It is important to note that both studies assessed emotion dynamics on one timescale over seven days. Yet, numerous recent studies have highlighted the importance of assessing whether dynamical phenomenon differ based on the timescale of their measurement (moment-to-moment, day-to-day, weekday vs. weekend, etc.) and that the timescale of measurement can lead to differential conclusions regarding associations with other variables of interest (e.g., treatment outcome; brain functioning; emotion regulation (Fisher \& Bosley, 2019; Jahng et al., 2008; 
Kuppens, 2015; Liégeois et al., 2019; Oravecz \& Brick, 2019; Stange et al., 2019). Furthermore, the extent to which emotion dynamics reflect stable trait-like variation in emotions is still unclear (Wendt et al., 2019). Thus, the importance of studying the stability of these findings across different timescales is an essential next step.

\section{Goals and Hypotheses}

The present study aimed to replicate findings from Sperry and Kwapil (2019) and Sperry, Walsh, and Kwapil (2019) and extend these findings in four important ways: 1) we expanded our ESM protocol to comprehensively examine high and low arousal NA and PA as well as unpleasant and pleasant experiences and events, 2) we controlled for mean levels of NA, PA, depression, and neuroticism to show that emotion dynamics are a unique aspect of the emotional experience associated with bipolar spectrum psychopathology, 3) we expanded the sampling period to 14 days in order to increase power to examine within and between day differences in

emotion dynamics, and 4) we did so across a wide spectrum of bipolar psychopathology enriched with high scorers on the HPS. The following a priori hypotheses were pre-registered on Open Science Framework (http://osf.io/xjnfd).

We predicted that bipolar spectrum psychopathology, as measured by the HPS, would be associated with 1) hyper-reactivity of high and low arousal NA and high arousal PA, 2) elevated variability and instability of high arousal NA and PA, 3) large increases in NA and PA, and 4) PA inertia within, but not between-days. We predicted that bipolar spectrum psychopathology would be associated with these patterns of emotion dynamics over-and-above mean levels of NA and PA, neuroticism, and current depressive symptoms. Hypotheses regarding within versus between-day dynamics were exploratory (with the exception of inertia hypotheses) given the lack 
of previous investigations of short-term dynamics and differing timescales in bipolar spectrum psychopathology.

\section{Methods}

\section{Power Analysis}

Monte Carlo Simulation is the most suitable method for determining power in multilevel data (Heck \& Thomas, 2015; Muthén \& Muthén, 2010). Using 135 participants from preliminary findings (Sperry \& Kwapil, 2019), we estimated two models using assumed population values (produced by saving model estimations based on pilot data), entering all parameters, and specifying 500 replications. For 135 participants with an average of 37 observations nested within person, power was estimated at 0.80 for detecting direct effects and .69 for cross-level interactions. Sample sizes for Pearson correlations and linear regressions were determined using G*Power (Faul, Erdfelder, Lang, \& Buchner, 2007). For a bivariate normal correlation, a sample size of 193 individuals was sufficient to detect small effects with $80 \%$ power. We calculated sample size for linear regressions based on $R^{2}$ increase in a three-step model with three predictors - suggesting a sample size of 247. Based on previous studies (Sperry \& Kwapil, 2017; Sperry \& Kwapil, 2019) we anticipated having to drop $10 \%$ of the sample. Thus, the final recruitment goal was a minimum of 274 participants.

\section{Participants}

A total of 352 undergraduate students were enrolled into the study via two methods. First, any participants in the course-credit subject pool were able to sign up for the study. Second, participants who scored at least 1.5 standard deviations above the mean on the HPS screening items that were included in the departmental pre-screening questionnaire were invited to participate. This recruitment method has been successfully used before to ensure adequate 
inclusion of high scoring participants. Undergraduates have been widely used in studies of bipolar spectrum psychopathology and are appropriate in that they are just entering into the period of greatest risk for developing bipolar disorders. Additionally, college students with elevated scores on the HPS report bipolar symptoms and have elevated risk for bipolar disorders (Kwapil et al., 2000; Walsh et al., 2015).

The final sample included 233 participants with usable data. Participants were excluded from analyses $(n=119)$ due to technical difficulties with ESM data collection $(n=49)$, too few ESM questionnaires ( $<20$ per week; $n=61)$, withdrawal due to time constraints $(n=5)$, and invalid responding $(n=4)$. Exclusion criteria regarding number of completed ESM questionnaires was based on two factors. First, the Hox ratio defines adequate power to detect intraindividual variability as at least 20 observations nested within the individual (Hox, 2002). Importantly, planned missing-data monte carlo simulations show minimal bias on parameter estimates at the floor of 20 questionnaires (Silvia, Kwapil, Walsh, \& Myin-Germeys, 2014). Second, in order to compute indices of instability, it is important to have enough ESM questionnaires per day to adequately assess successive differences. Note that there was a larger rate of exclusions than proposed in the pre-registration ${ }^{1}$. Participants received course credit and those who completed at least $70 \%$ of the ESM questionnaires were entered into a drawing for a $\$ 100$ gift card. The study was approved by the Institutional Review Board and all participants signed informed consent.

\section{Materials}

\footnotetext{
${ }^{1}$ This rate appears to be driven by two factors. First, the ESM application Expimetrics failed to work on smartphones purchased outside of the United States. The second issue arose from a decline in completed questionnaires during the second week of participation. Note that there were no significant differences between those included and those excluded on any lab measures or demographics (Table 1), with the exception of non-native English speakers and time living in the United States (presumably directly related to the international smartphone issue).
} 
Participants completed self-report measures of bipolar spectrum psychopathology, infrequent responding, anhedonic depression, neuroticism, and demographics. The HPS is a 48-item truefalse questionnaire that assesses bipolar spectrum psychopathology. High HPS scores are uniquely associated with bipolar spectrum psychopathology (e.g., Walsh et al., 2015), but not with other conditions involving prominent emotional disturbances such as unipolar depression or borderline personality disorder. The HPS was intermixed with a 13-item infrequency scale (Chapman \& Chapman, 1983) designed to detect invalid protocols. Following Chapman and Chapman, participants who endorsed more than two infrequency items were excluded from the analyses. Current levels of depressive symptoms were measured using the 8-item anhedonic depression subscale from the Mood and Anxiety Symptoms Questionnaire (MASQ-AD; Watson, Clark, et al., 1995; Watson, Weber, et al., 1995). The MASQ-AD taps depressed mood, lack of motivation, and other symptoms of depression experienced during the past week (Nitschke, Heller, Imig, McDonald, \& Miller, 2001; Watson, Clark, et al., 1995; Watson, Weber, et al., 1995). We used a 7-item version that excluded the item assessing suicidality based on requirements from the institutional review board. The 12-item Neuroticism subscale of the NEOFive Factor Inventory (McCrae \& Costa, 2010) was administered to measure neuroticism.

Experience Sampling Methodology Questionnaire. The ESM protocol included 24 items that tapped emotions, daily experiences, and cognitive and behavioral manifestations of bipolar spectrum psychopathology (Table S1). Emotion items were drawn from the affective circumplex (Russell, 1980) and Positive and Negative Affect Scale (Watson, Clark, \& Tellegen, 1988) to include high and low arousal NA and high and low arousal PA items. Unpleasant and pleasant experience items were based on previous research examining affective reactivity using ESM (Myin-Germeys et al., 2003; Pishva et al., 2014). All items were scored on a 7-point Likert 
scale ranging from " 1 : Not at all” to "7: Very much." We created five indices using the ESM data: high arousal NA (NAHA), low arousal NA (NA $\left.\mathrm{NA}_{\mathrm{HA}}\right)$, high arousal PA (PAHA), low arousal PA (PALA), impulsivity.

\section{Procedures}

Participants first completed a one-hour information session during which they were administered the self-report questionnaires and instructed on ESM procedures using either the ESM application Metricwire or Expimetrics on their smartphone. Participants completed a full practice trial before leaving the lab (not included in analyses). We switched from Metricwire to Expimetrics mid-study; however, participants' experience of completing surveys on Metricwire and Expimetrics was largely identical. Participants completed ESM questionnaires for 14 days in their normal daily environment. They were prompted eight times daily between $10 \mathrm{am}$ and $10 \mathrm{pm}$ at random times within stratified 90 minutes intervals. Participants were instructed to begin the surveys within 10 minutes of the signal, after which the link to the survey expired, ensuring that participants could not skip questionnaires and complete them later. The ESM questionnaire required approximately two minutes to complete. Participants returned to the lab for two followup sessions, mid-way through each week of participation. These follow-up sessions are a standard part of our procedures and are included to motivate participation, ensure appropriate practices, and troubleshoot problems.

\section{Statistical Analyses}

ESM data has a hierarchical structure in which ratings in daily life (level 1 data) are nested within participants (level 2 data). In some cases, ESM data have three levels (ESM ratings nested within days nested within participants). Hierarchical linear modeling is recommended for ESM data as it provides a more appropriate method of analyzing nested data than conventional 
unilevel analyses (Nezlek, 2012). Level 1 predictors were group mean centered and level 2 predictors were grand mean centered. Note that data from the first ESM protocol of each day was not included in the computations of instability and inertia so that indices did not include overnight lags.

In order to assess affective reactivity, cross-level interactions were conducted to test whether the level 2 predictor (HPS) was associated with the slope of the level 1 predictor and criterion (e.g., the slope of situation unpleasant predicting $\mathrm{NA}_{\mathrm{HA}}$ in the moment) controlling for the level 1 criterion at the prior moment. Equation 1 represents the examination of affective reactivity with HPS scores controlling for emotion at the prior moment:

\section{(Insert Equation 1)}

In order to examine inertia, a lag variable was created that represented $\mathrm{PA}_{t+1}$. An autoregressive slope model was then used to examine whether HPS scores predicted the slope between $\mathrm{PA}_{t}$ and $\mathrm{PA}_{t+1}$. Affective variability of NA and PA was computed using within-person variance (WPV), which involves computing the standard deviation of an individual's PA and NA items across days and between days (Eid \& Diener, 1999; Jahng et al., 2008). Within-day WPV was calculated by taking the SD of all responses over the 14-day protocol. This produces one value per person and was regressed on HPS scores. To calculate between-day WPV, SD's were calculated for each day which produced 14 nested WPV values (one per day). Thus, for betweenday analyses we examined the direct effect between WPV values (at level 1) and HPS scores (at level 2).

Two measures of affective instability were computed: adjusted square of successive differences (ASSD) and probability of acute change (PAC). Three steps were taken to compute ASSD. First, lag variables were created for PA, NA, and time between signals (ESM 
questionnaires sent). Second, we calculated the square of successive differences of PA and NA, (i.e., the squared differences between time $t$ and time $t_{t+1}$ ). Given that some participants had significantly longer lags than others due to missed questionnaires, we set any lag greater than 6 hours to missing. Following Jahng et al. (2008), we adjusted squared successive differences to create ASSD by dividing the squared successive differences by $\left[\left(t_{i+1}-t_{i}\right) / M d n\left(t_{i+1}-t_{i}\right)\right]^{\lambda}$ where $M d n\left(t_{i+1}-t_{i}\right)$ was the median of the time intervals for all is for each individual. Lambda $(\lambda)$ was chosen to make successive differences as constant as possible.

PAC also reflects affective instability but is the number of acute changes in an emotion divided by the number of total changes across occasions (Jahng et al., 2008). PAC enables us to examine the likelihood of a successive large increase in NA or PA. PAC was calculated via Equation 2: (Insert Equation 2)

where $\mathrm{AC}_{i+1} \mathrm{AC}_{\mathrm{i}+1}=1$, if $\mathrm{x}_{\mathrm{i}+1}-\mathrm{x}_{\mathrm{i}} \geq c$ and $\mathrm{AC}_{\mathrm{i}+1}=0$, if $\mathrm{x}_{\mathrm{i}+1}-\mathrm{x}_{\mathrm{i}} \leq c$. Note that $\mathrm{c}$ represents a predetermined cutoff that can be determined theoretically or statistically. We drew from studies of borderline personality disorder and preliminary studies of bipolar spectrum psychopathology which use AC cutoff at the $90^{\text {th }}$ percentile (Jahng et al., 2008; Sperry \& Kwapil, 2019; Sperry, Walsh, \& Kwapil, 2019).

\section{Results}

Recruitment of high scorers was successful in that the mean score on the HPS (Table 1) was consistent with or higher than other samples that used similar enrichment procedures (e.g., Walsh et al., 2012; Walsh et al., 2015; Sperry \& Kwapil, 2017). Notably, Walsh et al. (2015) reported that $40 \%$ of those in the third quartile and $58 \%$ of participants upper quartile of HPS scores met criteria for a bipolar spectrum disorder. Twenty-six percent $(n=60)$ of the current sample had HPS scores that fell into the upper quartile (27 to 48) indicating that we adequately 
captured high scorers who have a high likelihood of a diagnosable bipolar spectrum disorder or a history of hypomanic episodes.

Participants completed an average of 35 usable ESM protocols $(\mathrm{SD}=7.11$, range $=20$ $52, \%$ completion $=62.5)$ during the first week and 34 during the second week $(\mathrm{SD}=8.12$, range $=20-53, \%$ completion $=60.7)$ resulting in 16,202 total level 1 observations. Total number of completed ESM questionnaires was unassociated with HPS scores $(r=0.04, p=.60)$, anhedonic depression $(r=-.03, p=.65)$, or neuroticism $(r=-.02, p=.80)$. Descriptive statistics and correlations of emotion dynamics are presented in Supplemental Table 2. Direct effects between HPS scores and ESM items are presented in Table 2. Supplemental Figures 1 and 2 display the time-series for NA and PA, respectively, for a subset of participants who completed at least $75 \%$ of total ESM questionnaires sent (range 85-112 questionnaires) revealing marked individual differences. Figure 1 displays two randomly chosen participants who show heightened within and between-day instability of NA and between-day instability of PA.

The HPS (coefficient alpha $=.85)$, NEO-FFI Neuroticism (coefficient alpha $=.82)$, and the MASQ Anhedonic Depression (coefficient alpha $=.84$ ) all had good internal reliability. The HPS was unassociated with both neuroticism $(r=.12, p=.07)$ and anhedonic depression $(r=$ $.12, p=.23)$. Anhedonic depression and neuroticism were correlated with a medium effect size $(r$ $=.48, p<.001)$.

\section{Affective Reactivity}

Eight multilevel models were run assessing reactivity to stressful situations, unpleasant events, enjoying one's activity, and pleasant events (Table 3). Participants were more likely to report NAHA and NA:LA as levels of stress increased and they engaged in unpleasant events; however, contrary to expectation, this was not moderated by HPS scores. We also tested whether 
HPS scores moderated the association between stress/unpleasant events and feeling like one's emotions are out of control. Participants were more likely to report feeling like their emotions were out of control as stress levels increased and as they experienced unpleasant events. HPS scores moderated this association such that when experiencing a stressful situation or unpleasant event, those high on the HPS were more likely to report their emotions were out of control compared to low scorers (see Figure 2). In terms of positive affect reactivity, we tested whether HPS scores moderated the association between enjoying one's activity or reporting a pleasant event with $\mathrm{PA}_{\mathrm{HA}}$. Overall, participants are more likely to report $\mathrm{PA}_{\mathrm{HA}}$ when they were enjoying their activity more or engaging in a pleasant event. This was further moderated by HPS scores such that when reporting that they were enjoying their activity or engaged in a pleasant event, those high in bipolar spectrum psychopathology were more likely to experience PAHA compared to low scorers (see Figure 3).

\section{Affective inertia}

Contrary to expectations, bipolar spectrum psychopathology was unassociated with PA inertia. Specifically, HPS scores did not moderate the lagged association of high arousal $\mathrm{PA}_{t}$ and high arousal $\mathrm{PA}_{t-1}(Y=.002, \mathrm{SE}=.015, p=.88)$.

\section{Affective variability}

First, we ran four linear regressions to test whether within-day variability was associated with HPS scores (Table 4). HPS scores were associated with within-day variability of NA $\mathrm{HA}_{\text {and }}$ NA $A_{L A}$ over-and-above mean levels of $\mathrm{NA}_{\mathrm{HA}}$ and NA $\mathrm{NA}_{\text {LA }}$ but were unassociated with variability of $\mathrm{PA}_{\mathrm{HA}}$ or $\mathrm{PA}_{\mathrm{LA}}$. Second, we ran four multilevel models to test whether between-day variability was associated with HPS scores (Table 5). HPS scores were associated with between-day variability of $\mathrm{NA}_{\mathrm{HA}}, \mathrm{NA}_{\mathrm{LA}}$, and $\mathrm{PA}_{\mathrm{HA}}$ over-and-above day mean levels of those emotions but was 
unassociated with $\mathrm{PA}_{\mathrm{LA}}$. These results remained after accounting for neuroticism and anhedonic depression (see Tables S3 \& S4).

\section{Affective instability ${ }^{2}$}

First, we ran four multilevel models to test whether within-day instability (as measured by ASSD) was associated with HPS scores (Table 4). HPS scores were associated with withinday instability of $\mathrm{NA}_{\mathrm{HA}}, \mathrm{NA}_{\mathrm{LA}}$, and $\mathrm{PA}_{\mathrm{HA}}$ over-and-above mean levels of those emotions. Second, we ran four multilevel models to test whether between-day instability (ASSD) was associated with HPS scores (Table 5). HPS scores were associated with between-day instability of $\mathrm{NA}_{\mathrm{HA}}, \mathrm{NA}_{\mathrm{LA}}$, and $\mathrm{PA}_{\mathrm{HA}}$ over-and-above day mean levels of those emotions. Note that HPS scores were unassociated with both within-day and between-day instability of PALA. These results remained after accounting for neuroticism and anhedonic depression (Tables S3 \& S4). Next, we examined whether HPS scores were associated with large increases in NA or PA, as measured by PAC. We ran four multilevel models to test within-day PAC (Table 4). HPS scores were associated with within-day PAC of NA $\mathrm{LA}_{\text {a }}$ but not NAHA, PAHA, or PALA. We then ran four multilevel models to test between-day PAC (Table 5). HPS scores were associated with between-day PAC of $\mathrm{NA}_{\mathrm{LA}}$ and $\mathrm{PA}_{\mathrm{NA}}$ but not $\mathrm{NA}_{\mathrm{HA}}$ or $\mathrm{PA}_{\mathrm{LA}}$. All results remained after accounting for neuroticism and anhedonic depression (Tables S3 \& S4). A summary of all associations between HPS scores and within- and between-day emotion dynamics are presented in Table S5.

\section{Discussion}

This was the first study to our knowledge that comprehensively examined emotion dynamics as they relate to bipolar spectrum psychopathology across multiple timescales. The present

\footnotetext{
${ }^{2}$ Total level 1 observations dropped to 12,800 in the calculation of successive differences ( $79 \%$ of total observations) due to missing data and the deletion of overnight lags.
} 
findings, which build upon preliminary work by Sperry and Kwapil (2019) and Sperry, Walsh, and Kwapil (2019), provide strong evidence that bipolar spectrum psychopathology, even in a non-clinically ascertained sample, is associated with affective lability both moment-to-moment within-day and between-days. Furthermore, altered emotion dynamics were associated with bipolar spectrum psychopathology over-and-above mean levels of emotions in daily life, depressive symptoms, and neuroticism.

Overall, bipolar spectrum psychopathology was associated with dysregulated NA both within-and between-days. Consistent with our hypotheses, HPS scores were associated with variability and instability of $\mathrm{NA}_{\mathrm{HA}}$ and $\mathrm{NA}_{\mathrm{LA}}$ over-and-above mean levels of NA, anhedonic depression, and neuroticism. These findings are consistent with prior longer-term studies which reported non-linearity in mood symptoms in those with bipolar I disorder (Bonsall et al., 2012). As such, individuals high in bipolar spectrum psychopathology appear to experience frequent shifts in NA and depressive symptoms across multiple timescales. However, there were several hypotheses related to NA that were not supported. First, Sperry and Kwapil (2019) reported that over 7 days, HPS scores were associated with PAC of NAHA - in the present study HPS scores were only associated with PAC of NALA. This indicates that bipolar spectrum psychopathology, over a longer sampling period, seems to be specifically associated with large moment-to-moment increases in emotions such as sadness, boredom, and sluggishness but not anger, nervousness, or irritability. Future studies may benefit from examining PAC of discrete negative emotions such as sadness, anger, and irritability, rather than examining collapsed indices in an effort to disentangle whether bipolar spectrum psychopathology is associated with large moment-tomoment increases in high and low arousal emotions. Second, HPS scores did not moderate the association of stress/unpleasant activity and NAHA. Although Sperry and Kwapil (2019) reported 
that HPS scores moderated the association of $\mathrm{NA}_{\mathrm{HA}}$ and experiencing a negative (rather than unpleasant) event, they did not control for lagged $\mathrm{NA}_{\mathrm{HA}}$ - this may partially explain why they did not see a replication in the present study. However, Sperry and Kwapil $(2017,2019)$ also did not find NA hyper-reactivity to stress. In contrast, the present study replicated Sperry and Kwapil (2019)'s findings that in the face of unpleasant or stressful experiences, those high in bipolar spectrum psychopathology are more likely to feel like their emotions are out of control. Thus, it seems that rather than reacting with an increase in NA, those high in bipolar spectrum psychopathology feel less control of their emotions in the face of distress. This may be in part due to poor emotion regulation those with bipolar disorder attempt to use emotion regulation strategies at equal rates but are less successful in doing so (Gruber, Harvey, \& Gross, 2012).

Contrary to expectation, HPS scores were unassociated with PA inertia, suggesting that individuals high in bipolar spectrum psychopathology are not characterized by a tendency to persist in terms of PA. However, this finding is consistent with a study that reported no differences in affective recovery following negative or positive events (Gruber, Harvey, \& Purcell, 2011). Rather, in the present sample, bipolar spectrum psychopathology was associated with elevated mean-levels of $\mathrm{PA}_{\mathrm{HA}}$, reactivity in response to enjoyable activities and pleasant activities, and instability of PAHA both within- and between-days. Associations between HPS scores and variability and PAC differed based on timescale. Specifically, HPS scores were associated with high variability of $\mathrm{PA}_{\mathrm{HA}}$ between- but not within-days suggesting that people high in bipolar spectrum psychopathology consistently stay around their mean level of PAHA over the course of a day; however, there is little consistency in how much their PA deviates from core affect between days. Similarly, HPS scores were associated with large moment-to-moment increases in PAHA between but not within days. HPS scores were unassociated with any 
dynamics related to $\mathrm{PA}_{\mathrm{HA}}$. Taken together, this suggests that dysregulation of PA may be specific to a) timescale and b) high arousal PA. Importantly, dysregulated PA, an understudied area, has also been linked to decreased well-being, depression, and anxiety (Gruber, Kogan, Quoidbach, \& Mauss, 2013).

Prominent theoretical models of emotion help elucidate the connection between altered emotion dynamics and bipolar spectrum psychopathology. The DynAffect Model, proposed by Kuppens, Oravecz, and Tuerlinckx (2010), posits that emotion dynamics reflect three primary processes that are unique to each individual: attractor home base, attractor strength, and variability. Attractor home base represents "core" affect or set point/baseline affect. This system is proposed to keep individuals balanced by returning affect to baseline following reactivity. Attractor strength represents the extent to which the individual deviates away from their homebase. Lastly, individuals experience many internal and external events that are represented by stochastic variability in the shifts to and away from the attractor home base. Importantly, these three processes can function differently across levels of valence and arousal. In light of this theoretical model, the present findings raise three issues regarding emotion dynamics in bipolar spectrum psychopathology. First, bipolar spectrum psychopathology may be represented by two distinct attractor home bases - one that is high negative valence and high arousal and the other that is high positive valence and high arousal. This is evidenced by both high mean levels of high arousal (but not low arousal) NA and PA in daily life. It is unclear whether these home bases are simultaneously activated or whether they switch on and off depending on context. This would be important to understand as several studies suggest that experiencing relatively more positive than negative emotions is important to psychological adjustment (Diener, Oishi, \& Lucas, 2003; Fredrickson \& Losada, 2005) and emotional stability (Kuppens, Van Mechelen, Nezlek, 
Dossche, \& Timmermans, 2007). Studies on affective bipolarity, or the extent to which individuals experience NA and PA as orthogonal or opposite ends of one spectrum (Dejonckheere et al., 2018), may help elucidate how these two potential home-bases function together or separately. Second, bipolar spectrum psychopathology may be characterized by aspects of low attractor strength. This is represented by high variability (WPV) of NA and PA as individuals tend to deviate from their home base and are hyper-reactive to stressful and pleasant events. However, consistent with prior studies (Sperry \& Kwapil, 2019; Sperry, Walsh, \& Kwapil, 2019), participants were not characterized by inertia, which indicates that they are able to return to their home base despite large deviations and reactivity in PA. It would be important to establish whether those with bipolar disorders have very low attractor strength in which they have large deviations from their home base with those extremes persisting during mood episodes. It may be that what separates euthymia from bipolar mood episodes is the ability of the individual to return to home base following perturbations in emotions. Lastly, bipolar spectrum psychopathology is characterized by high variability - individuals deviate from their home base frequently. This aspect of the DynAffect Model can be represented by instability (MSSD) as it reflects some temporal dependency.

Having a high positive valence/high arousal attractor home base in combination with low attractor strength and high instability may be particularly problematic for individuals at risk for developing bipolar disorders. An attractor home base characterized by slight positive valence and arousal is proposed to help motivate people to approach, learn, and explore (Cacioppo \& Gardner, 1999). Individuals with bipolar disorder may have "faulty" home base systems whereby they experience extremes of valence and arousal that impair other aspects of affective functioning (i.e., resulting in reactivity and instability) and produce excessive approach 
behaviors or poor constraint (resulting in impulsivity; Sperry, 2016; Urosevíc, Abramson, Harmon-Jones, \& Alloy, 2008). Altered emotion dynamics (high instability and variability), especially in the positive valence domain, may reflect a breakdown of the feedback systems between emotion, behavior, and cognition proposed by prominent theories of emotion (Fridja, 2007; Frijda \& Scherer, 2009; Scherer, 2009).

\section{Limitations and Future Directions}

Although initial studies suggested that emotion dynamics provide useful and unique information about affect in daily life, their measurement has increasingly been criticized. Most notably, studies have largely failed to examine emotion dynamics over-and-above mean levels of affect. However, in both preliminary studies (Sperry \& Kwapil, 2019) and the current study, HPS scores were associated with emotion dynamics over-and-above mean affect with little attenuation in their effects. This is relevant in light of concerns that mathematical computations of emotion dynamics are conflated or redundant with mean affect (Baird, Le, \& Lucas, 2006; Dejonckheere et al., 2019). and the current study, HPS scores were associated with emotion dynamics overand-above mean affect with little attenuation in their effects. This is relevant in light of concerns that mathematical computations of emotion dynamics are conflated or redundant with mean affect (Dejonckheere et al., 2019). The authors concluded that many indices of emotion dynamics are highly related and that not all dynamics need to be measured to have the best predictive model of psychopathology. Note that in the present study, we also found high correlations among same-valence, same-arousal emotion dynamics. Furthermore, we did not see much differentiation in patterns of associations with emotion dynamics - bipolar spectrum psychopathology was associated with elevated mean levels, WPV, MSSD, and PAC, but not inertia. However, for WPV and PAC, there were differences in timescale. Thus, future studies 
may be able to measure fewer emotion dynamics with the same amount of predictive power depending on the timescale of their measurements. Importantly, Dejonckheere et al. (2019) note that the most "important" dynamic assessed may differ between disorders. For example, depression is most highly associated with high mean levels of NA whereas borderline personality disorder is most highly associated with high variability of NA. Of note, in Sperry, Walsh, and Kwapil (2019), instability (ASSD) of NA and PA was the strongest predictor of bipolar symptoms and diagnoses three-years later. This highlights that the relative predictive contribution of dynamics, over-and-above mean levels, may depend both on the type of psychopathology of interest and whether dynamics and symptoms are measured cross-sectionally or longitudinally.

The computation of emotion dynamics relies on many time points. Missing data can be highly problematic for the modeling emotion dynamics that account for temporal dependency (Ebner-Priemer \& Trull, 2012). If too many ESM questionnaires are missed, successive differences cannot be meaningfully computed, and parameter estimates may be biased. Thus, a number of participants had to be excluded because they did not complete a sufficient amount of ESM questionnaires to adequately measure successive differences. An important advantage of the present study is that we assessed daily life experiences for two weeks, rather than one week typical of previous studies (e.g., Sperry \& Kwapil, 2019). However, compliance decreased in the second week of participation such that a number of participants were dropped who had adequate compliance in the first week, but not the second week. Future studies need to weigh the pros and cons of how frequently to sample participants based on the phenomenon of interest, the expected burden of the study, and the inclusion of incentives to motivate participation. 
The present study is based on participants with a broad range of scores on the HPS, presumably representing a range of bipolar spectrum psychopathology. Two issues should be considered. First of all, it is likely that some of the participants already have experienced diagnosable bipolar disorders, despite their relatively young age (although this was not assessed in the present study). Eckblad and Chapman (1986) reported that $78 \%$ of their high HPS-scoring college students met criteria for a hypomanic episode (although not all of them had diagnosable bipolar disorders). In Walsh et al.'s, (2012) cross-sectional study of college students, $22 \%$ of participants scoring at least 1.5 SD above the mean on the HPS met criteria for DSM bipolar disorders and 35\% met criteria for broad bipolar spectrum disorders. Thus, some of our participants likely had already experienced manic or hypomanic episodes, or possibly were in such states during the study. Conversely, it is worth considering that not all of the high scorers on the HPS are expected to develop bipolar spectrum disorders. However, note that in a threeyear follow-up assessment, Walsh et al. (2015) reported that among their upper quartile of HPS scorers, $27 \%$ met criteria for a DSM-IV-TR bipolar disorder, $58 \%$ met criteria for a broad bipolar spectrum disorder, and $81 \%$ met criteria for hyperthymic temperament or a hypomanic episode, although they did not have elevated rates of unipolar depressive disorders. Based on Walsh et al's statistics $(2012,2015)$, we can estimate that approximately $15 \%$ of our sample would likely meet criteria for a bipolar spectrum disorder and 20\% would likely meet criteria for having had a hypomanic episode. Furthermore, this sample appeared to be experiencing some symptoms of anhedonic depression. The mean score on the anhedonic subscale of the MASQ was roughly $1 \mathrm{SD}$ above means published by Bredemeier et al. (2010). Importantly, a mean score of 20 on the anhedonic subscale had generally good sensitivity and specificity in predicting 
current depressive episodes (sensitivity $=.80-1.00$; specificity $.73-.87$; positive predictive value $=.10-.16$; negative predictive power $=.99-1.00)$.

Nevertheless, it is unclear the extent to which these dynamics generalize to clinically diagnosed patients. When considering emotion dynamics across the full spectrum of bipolar psychopathology, future studies should examine whether emotion dynamics a) are stable over time, b) change episodically, and c) whether the intensity of disruptions differ based on severity of psychopathology. It is important to note that increasing studies suggest that college student samples are not generalizable to community samples; however, epidemiological research also suggests that college students have higher rates of psychopathology than the general population which suggests that we can capture the phenomenon we are interested in in this population (Auerbach et al., 2018). None-the-less, these findings should be replicated in community samples.

\section{Clinical Implications}

The assessment of emotion dynamics in daily life is essential for optimizing moodmonitoring interventions in bipolar disorder and for ideographically tailoring intervention to clients. In fact, several studies have noted the importance of examining individual differences in the temporal dynamics of mood in terms of both tailor treatments (e.g., Fisher \& Boswell, 2016) and assessing treatment response (e.g., Fisher \& Newman, 2016). For example, if a patient undergoes an assessment prior to engaging in therapy and displays an emotional pattern high in instability, variability, and PAC, they may particularly benefit from an intervention that specifically targets emotion regulation and distress tolerance (e.g., Dialectical Behavior Therapy). In contrast, a patient who shows an inflexible pattern of emotional responding (e.g., high inertia, low variance), may benefit from emotional awareness training or interoceptive 
exposure to strong emptions. Then, throughout treatment, mood monitoring could be used to assess the efficacy of the intervention. The present study also highlights several important implications for this type of work going forward. Given that those high in bipolar spectrum psychopathology experience within- and between-day instability, mood monitoring interventions need to sample emotions frequently enough to assess change in emotion states over relatively short periods of time. However, it will be important to complete longer studies (e.g., a month or longer), or repeated shorter assessments using measurement-burst designs, to capture the stability of emotion dynamics in daily life and determine the feasibility of long-term mood-monitoring.

\section{Conclusions}

This study, in combination with preliminary studies (Sperry \& Kwapil, 2019; Sperry, Walsh, \& Kwapil, 2019), provides evidence that bipolar spectrum psychopathology is associated with lability of both NA and PA on a day-to-day basis even outside of the context of mood episodes. Inter-episodic and prodromal emotion dysregulation may be a particularly important risk factor for the development of bipolar spectrum disorders and treatment target for those with clinical impairment. Furthermore, using ambulatory assessments to measure the real-world expression of bipolar spectrum psychopathology provides a novel and ecologically valid way of assessing emotion dynamics and should enhance our ability to develop sophisticated mood monitoring applications for bipolar spectrum disorders.

\section{References}

Ahmed, A. O., Green, B. A., Clark, C. B., Stahl, K. C., \& McFarland, M. E. (2011). Latent structure of unipolar and bipolar mood symptoms. Bipolar Disorders, 13(5-6), 522-536. https://doi.org/10.1111/j.1399-5618.2011.00940.x

Alloy, L. B., Abramson, L. Y., Walshaw, P. D., Keyser, J., \& Gerstein, R. K. (2006). A cognitive 
vulnerability-stress perspective on bipolar spectrum disorders in a normative adolescent brain, cognitive, and emotional development context. Development and Psychopathology, 18(4), 1055-1103. https://doi.org/10.1017/S0954579406060524

Angst, J., Gamma, A., Benazzi, F., Ajdacic, V., Eich, D., \& Rössler, W. (2003). Toward a redefinition of subthreshold bipolarity: epidemiology and proposed criteria for bipolar-II, minor bipolar disorders and hypomania. Journal of Affective Disorders, 73(1-2), 133146. https://doi.org/10.1016/S0165-0327(02)00322-1

Angst, J., Gamma, A., \& Endrass, J. (2003). Risk factors for the bipolar and depression spectra. Acta Psychiatrica Scandinavica, 108(s418), 15-19. https://doi.org/10.1034/j.16000447.108.s418.4.x

Auerbach, R. P., Mortier, P., Bruffaerts, R., Alonso, J., Benjet, C., Cuijpers, P., ... Hasking, P. (2018). WHO World Mental Health Surveys International College Student Project: prevalence and distribution of mental disorders. Journal of Abnormal Psychology, $127(7), 623$.

Baird, B. M., Le, K., \& Lucas, R. E. (2006). On the nature of intraindividual personality variability: Reliability, validity, and associations with well-being. Journal of Personality and Social Psychology, 90(3), 512.

Bonsall, M. B., Wallace-Hadrill, S. M. ., Geddes, J. ., Goodwin, G. ., \& Holmes, E. . (2012). Nonlinear time-series approaches in characterizing mood stability and mood instability in bipolar disorder. Proc. R. Soc. B, 279(1730), 916-924.

Bredemeier, K., Spielberg, J. M., Silton, R. L., Berenbaum, H., Heller, W., \& Miller, G. A. (2010). Screening for depressive disorders using the Mood and Anxiety Symptoms 
Questionnaire Anhedonic Depression Scale: a receiver-operating characteristic analysis. Psychological Assessment, 22(3), 702-710. https://doi.org/10.1037/a0019915

Cacioppo, J. T., \& Gardner, W. L. (1999). Emotion. Annual Review of Psychology, 50(1), 191214. https://doi.org/10.1146/annurev.psych.50.1.191

Cochran, A. L., McInnis, M. G., \& Forger, D. B. (2016). Data-driven classification of bipolar I disorder from longitudinal course of mood. Translational Psychiatry, 6(10), e912-e912. https://doi.org/10.1038/tp.2016.166

Dejonckheere, E., Mestdagh, M., Houben, M., Erbas, Y., Pe, M., Koval, P., ... Kuppens, P. (2018). The bipolarity of affect and depressive symptoms. Journal of Personality and Social Psychology, 114(2), 323.

Dejonckheere, E., Mestdagh, M., Houben, M., Rutten, I., Sels, L., Kuppens, P., \& Tuerlinckx, F. (2019a). Complex affect dynamics add limited information to the prediction of psychological well-being. Nature Human Behavior, 3, 478-491. https://doi.org/10.1038/s41562-019-0555-0

Diener, E., Oishi, S., \& Lucas, R. E. (2003). Personality, culture, and subjective well-being: Emotional and cognitive evaluations of life. Annual Review of Psychology, 54(1), 403425.

Dilsaver, S. C. (2011). An estimate of the minimum economic burden of bipolar I and II disorders in the United States: 2009. Journal of Affective Disorders, 129(1), 79-83.

Ebner-Priemer, U. W., \& Trull, T. J. (2012). Investigating temporal instability in psychological variables: Understanding the real world as time dependent. Handbook of Research Methods for Studying Daily Life, 423-439.

Eckblad, M., \& Chapman, L. J. (1986). Development and validation of a scale for hypomanic 
personality. Journal of Abnormal Psychology, 95(3), 214.

Eid, M., \& Diener, E. (1999). Intraindividual variability in affect: Reliability, validity, and personality correlates. Journal of Personality and Social Psychology, 76(4), 662.

Farmer, A., Lam, D., Sahakian, B., Roiser, J., Burke, A., O’neill, N., ... McGuffin, P. (2006). A pilot study of positive mood induction in euthymic bipolar subjects compared with healthy controls. Psychological Medicine, 36(9), 1213-1218.

Faul, F., Erdfelder, E., Lang, A.-G., \& Buchner, A. (2007). G* Power 3: A flexible statistical power analysis program for the social, behavioral, and biomedical sciences. Behavior Research Methods, 39(2), 175-191.

Fisher, A. J., \& Bosley, H. G. (2019, January 30). Identifying the Presence and Timing of Discrete Mood States Prior to Therapy. https://doi.org/10.31219/osf.io/2jrhf

Fisher, A. J., \& Boswell, J. F. (2016). Enhancing the personalization of psychotherapy with dynamic assessment and modeling. Assessment, 23(4), 496-506.

Fisher, A. J., \& Newman, M. G. (2016). Reductions in the diurnal rigidity of anxiety predict treatment outcome in cognitive behavioral therapy for generalized anxiety disorder. Behaviour Research and Therapy, 79, 46-55.

Fredrickson, B. L., \& Losada, M. F. (2005). Positive affect and the complex dynamics of human flourishing. American Psychologist, 60(7), 678.

Fridja, N. H. (2007). The Laws of Emotion. Mahway, NJ, US: Lawrence Erlbaum Associates Publishers.

Frijda, N. H., \& Scherer, K. R. (2009). Emotion definitions (psychological perspectives). In K. R. Sander \& K. R. Scherer (Eds.), The Oxford companion to emotion and the affective sciences (pp. 142-144). New York, NY: Oxford University Press. 
Gruber, J., Harvey, A. G., \& Gross, J. J. (2012). When trying is not enough: Emotion regulation and the effort-success gap in bipolar disorder. Emotion, 12(5), 997.

Gruber, J., Harvey, A. G., \& Purcell, A. (2011). What goes up can come down? A preliminary investigation of emotion reactivity and emotion recovery in bipolar disorder. Journal of Affective Disorders, 133(3), 457-466. https://doi.org/10.1016/j.jad.2011.05.009

Gruber, J., Kogan, A., Quoidbach, J., \& Mauss, I. B. (2013). Happiness is best kept stable: Positive emotion variability is associated with poorer psychological health. Emotion, 13(1), 1-6. http://doi.org/10/1037/a0030262

Heck, R. H., \& Thomas, S. L. (2015). An introduction ot multilevel modeling (Third Edit). New York, NY: Routledge.

Henry, C., Mitropoulou, V., New, A. S., Koenigsberg, H. W., Silverman, J., \& Siever, L. J. (2001). Affective instability and impulsivity in borderline personality and bipolar II disorders: similarities and differences. Journal of Psychiatric Research, 35(6), 307-312.

Henry, C., Van den Bulke, D., Bellivier, F., Roy, I., Swendsen, J., M’Baïlara, K., ... Leboyer, M. (2008). Affective lability and affect intensity as core dimensions of bipolar disorders during euthymic period. Psychiatry Research, 159(1), 1-6.

Hofmann, B. U., \& Meyer, T. D. (2006). Mood fluctuations in people putatively at risk for bipolar disorders. British Journal of Clinical Psychology, 45(1), 105-110.

Houben, M., Van Den Noortgate, W., \& Kuppens, P. (2015). The relation between short-term emotion dynamics and psychological well-being: A meta-analysis. Psychological Bulletin, 141(4), 901.

Hox, J. (2002). Quantitative methodology series. In Multilevel analysis techniques and applications. Marwah, NJ: Lawrence Erlbaum Associates Publishers. 
Jahng, S., Wood, P. K., \& Trull, T. J. (2008). Analysis of affective instability in ecological momentary assessment: Indices using successive difference and group comparison via multilevel modeling. Psychological Methods, 13, 354-375. https://doi.org/10.1037/a0014173

Judd, L. L., \& Akiskal, H. S. (2003). The prevalence and disability of bipolar spectrum disorders in the US population: re-analysis of the ECA database taking into account subthreshold cases. Journal of Affective Disorders, 73(1), 123-131.

Koval, P., Brose, A., Pe, M. L., Houben, M., Erbas, Y., Champagne, D., \& Kuppens, P. (2015). Emotional inertia and external events: The roles of exposure, reactivity, and recovery. Emotion, 15(5), 625.

Kuppens, P. (2015). It's About Time: A Special Section on Affect Dynamics. Emotion Review, 7(4), 297-300. https://doi.org/10.1177/1754073915590947

Kuppens, P., Allen, N. B., \& Sheeber, L. B. (2010). Emotional inertia and psychological maladjustment. Psychological Science, 21(7), 984-991.

Kuppens, P., Oravecz, Z., \& Tuerlinckx, F. (2010). Feelings change: Accounting for individual differences in the temporal dynamics of affect. Journal of Personality and Social Psychology, 99(6), 1042 - 1060.

Kuppens, P., Van Mechelen, I., Nezlek, J. B., Dossche, D., \& Timmermans, T. (2007). Individual differences in core affect variability and their relationship to personality and psychological adjustment. Emotion, 7(2), 262 - 274.

Kwapil, T. R., Barrantes-Vidal, N., Armistead, M. S., Hope, G. A., Brown, L. H., Silvia, P. J., \& Myin-Germeys, I. (2011). The expression of bipolar spectrum psychopathology in daily life. Journal of Affective Disorders, 130(1-2), 166-170. 
https://doi.org/10.1016/j.jad.2010.10.025

Kwapil, T. R., Miller, M. B., Zinser, M. C., Chapman, L. J., Chapman, J., \& Eckblad, M. (2000). A longitudinal study of high scorers on the Hypomanic Personality Scale. Journal of Abnormal Psychology, 109, 222-226. https://doi.org/10.1037/0021-843X.109.2.222

Liégeois, R., Li, J., Kong, R., Orban, C., Van De Ville, D., Ge, T., .. Yeo, B. T. T. (2019). Resting brain dynamics at different timescales capture distinct aspects of human behavior. Nature Communications, 10(1), 2317.

Lovejoy, M. C., \& Steuerwald, B. L. (1995). Subsyndromal unipolar and bipolar disorders: Comparisons on positive and negative affect. Journal of Abnormal Psychology, 104(2), 381.

M'Bailara, K., Demotes-Mainard, J., Swendsen, J., Mathieu, F., Leboyer, M., \& Henry, C. (2009). Emotional hyper-reactivity in normothymic bipolar patients. Bipolar Disorders, 11(1), 63-69.

McCrae, R. R., \& Costa, P. T. (2010). NEO Inventories For The NEO Personality Inventory-3 (NEO-PI-3), NEO Five-Factor Inventory-3 (NEO-FFI-3), NEO Personality InventoryRevised (NEO PI-R): Professional Manual. Lutz, FL: PAR.

Merikangas, K. R., Akiskal, H. S., Angst, J., Greenberg, P. E., Hirschfeld, R. M. A., Petukhova, M., \& Kessler, R. C. (2007). Lifetime and 12-month prevalence of bipolar spectrum disorder in the National Comorbidity Survey replication. Archives of General Psychiatry, 64(5), 543-552.

Muthén, L. K., \& Muthén, B. O. (2010). Mplus user’s guide. Los Angeles, CA: Muthén \& Muthén.

Myin-Germeys, I., Peeters, F., Havermans, R., Nicolson N, A., DeVries M, W., Delespaul, P., \& 
Van Os, J. (2003). Emotional reactivity to daily life stress in psychosis and affective disorder: an experience sampling study. Acta Psychiatrica Scandinavica, 107(2), 124131. https://doi.org/10.1034/j.1600-0447.2003.02025.x

Nezlek, J. B. (2012). Multilevel modeling analyses of diary-style data. In M. R. Mehl \& T. S. Conner (Eds.), Handbook of research methods for studying daily life (pp. 357-383). New York, NY: The Guilford Press.

Nitschke, J. B., Heller, W., Imig, J. C., McDonald, R. P., \& Miller, G. A. (2001). Distinguishing dimensions of anxiety and depression. Cognitive Therapy and Research, 25(1), 1-22.

Oravecz, Z., \& Brick, T. R. (2019). Associations Between Slow-and Fast-Timescale Indicators of Emotional Functioning. Social Psychological and Personality Science, 10(7), 864873.

Pishva, E., Drukker, M., Viechtbauer, W., Decoster, J., Collip, D., Van Winkel, R., ... Derom, C. (2014). Epigenetic genes and emotional reactivity to daily life events: a multi-step geneenvironment interaction study. PloS One, 9(6), e100935.

Prisciandaro, J. J., \& Tolliver, B. K. (2015). Evidence for the continuous latent structure of mania and depression in out-patients with bipolar disorder: Results from the Systematic Treatment Enhancement Program for Bipolar Disorder (STEP-BD). Psychological Medicine, 45(12), 2595-2603.

Prisciandaro, J. J., Tolliver, B. K., \& DeSantis, S. M. (2018). Identification and initial validation of empirically derived bipolar symptom states from a large longitudinal dataset: an application of hidden Markov modeling to the Systematic Treatment Enhancement Program for Bipolar Disorder (STEP-BD) study. Psychological Medicine, 49 (7), $1102-$ 1108. https://doi.org/10.1017/S0033291718002143 
Roshanaei-Moghaddam, B., \& Katon, W. (2009). Premature mortality from general medical illnesses among persons with bipolar disorder: a review. Psychiatric Services, 60(2), $147-156$.

Russell, J. A. (1980). A circumplex model of affect. Journal of Personality and Social Psychology, 39(6), 1161.

Silvia, P. J., Kwapil, T. R., Walsh, M. A., \& Myin-Germeys, I. (2014). Planned missing-data designs in experience-sampling research: Monte Carlo simulations of efficient designs for assessing within-person constructs. Behavior Research Methods, 46(1), 41-54. https://doi.org/10.3758/s13428-013-0353-y

Sperry, S. H. (2016). Examining the role of impulsivity in bipolar spectrum psychopathology: Identification and expression in daily life. Retrieved from https://libres.uncg.edu/ir/uncg/f/Sperry_uncg_0154M_11942.pdf

Sperry, S. H., \& Kwapil, T. R. (2017). What can daily life assessment tell us about the bipolar spectrum? Psychiatry Research, 252, 51-56. https://doi.org/10.1016/j.psychres.2017.02.045

Sperry, S. H., \& Kwapil, T. R. (2019). Affective Dynamics in Bipolar Spectrum Psychopathology: Modeling Inertia, Reactivity, Variability, and Instability in Daily Life. Journal of Affective Disorders, 251, 195-204. https://doi.org/doi.org/10.31234/osf.io/rcg93

Stange, J. P., Kleiman, E. M., Mermelstein, R. J., \& Trull, T. J. (2019). Using ambulatory assessment to measure dynamic risk processes in affective disorders. Journal of Affective Disorders, 259, 325-336. https://doi.org/10.1016/j.jad.2019.08.060

Steinacher, A., \& Wright, K. A. (2013). Relating the Bipolar Spectrum to Dysregulation of 
Behavioural Activation: A Perspective from Dynamical Modelling. PLoS ONE, 8(5), e63345. https://doi.org/10.1371/journal.pone.0063345

Szmulewicz, A. G., Martino, D. J., \& Strejilevich, S. A. (2019). Characterization of Mood Instability through Bipolar Disorders: A cluster-analytic approach using weekly prospective life-chart methodology. European Psychiatry, 57, 52-57. https://doi.org/10.1016/j.eurpsy.2018.10.003

Urosević, S., Abramson, L. Y., Harmon-Jones, E., \& Alloy, L. B. (2008). Dysregulation of the behavioral approach system (BAS) in bipolar spectrum disorders: review of theory and evidence. Clinical Psychology Review, 28(7), 1188-1205. https://doi.org/10.1016/j.cpr.2008.04.004

Walsh, M. A., DeGeorge, D. P., Barrantes-Vidal, N., \& Kwapil, T. R. (2015). A 3-year longitudinal study of risk for bipolar spectrum psychopathology. Journal of Abnormal Psychology, 124, 486-497. https://doi.org/10.1037/abn0000045

Walsh, M. A., Royal, A., Brown, L. H., Barrantes-Vidal, N., \& Kwapil, T. R. (2012). Looking for bipolar spectrum psychopathology: identification and expression in daily life. Comprehensive Psychiatry, 53(5), 409-421. https://doi.org/10.1016/J.COMPPSYCH.2011.06.006

Watson, D., Clark, L. A., \& Tellegen, A. (1988). Development and validation of brief measures of positive and negative affect: the PANAS scales. Journal of Personality and Social Psychology, 54(6), 1063.

Watson, D., Clark, L.A., Weber, K., Assenheimer, J.S., Strauss, M.E., \& McCormick, R.A. (1995). Testing a tripartite model: II. Exploring the symptom structure of anxiety and depression in student, adult, and patient samples. Journal of Abnormal Psychology, 
104(1), 15-25.

Watson, D., Weber, K., Assenheimer, J.S., Clark, L.A., Strauss, M.E., \& McCormick, R.A. (1995). Testing a tripartite model: I. Evaluating the convergence and discriminant validity of anxiety and depression symptom scales. Journal of Abnormal Psychology, 104(1), 3-14.

Wendt, L.P., Wright, A.G., Pilkonis, P.A., Woods, W.C., Denissen, J.J.A., Kühnel, A., \& Zimmermann, J. (2019, April 17). Indicators of affect dynamics: Structure, test-retest reliability, and personality correlates. http://doi/org/10.31234/osf.io/nyqst. 
Table 1. Demographic Characteristics and Descriptive Statistics

\begin{tabular}{|c|c|c|c|c|}
\hline & $\begin{array}{c}\text { Final Sample } \\
(n=233) \\
\text { Mean (SD) }\end{array}$ & $\begin{array}{c}\text { Dropped } \\
\text { sample } \\
(n=119) \\
\text { Mean (SD) }\end{array}$ & Test statistic & $p$ \\
\hline \multicolumn{5}{|l|}{ Demographics } \\
\hline Age (years) & $18.81(1.04)$ & $18.95(1.08)$ & $\mathrm{t}(350)=-1.20$ & .23 \\
\hline Time in US & $15.11(6.24)$ & $10.82(7.06)$ & $\mathrm{t}(83)=2.91$ & .005 \\
\hline Native English speaker (\%) & $87 \%$ & $76 \%$ & $X^{2}(1)=6.82$ & .009 \\
\hline \multicolumn{5}{|l|}{ Race $(\%)$} \\
\hline Black & $13 \%$ & $7 \%$ & & \\
\hline Asian & $22 \%$ & $31 \%$ & & \\
\hline White & $52 \%$ & $49 \%$ & $X^{2}(5)=5.93$ & .31 \\
\hline American Indian & $1 \%$ & $1 \%$ & & \\
\hline Native Hawaiian & $1 \%$ & $1 \%$ & & \\
\hline Multiracial & $11 \%$ & $11 \%$ & & \\
\hline \multicolumn{5}{|l|}{ Ethnicity $(\%)$} \\
\hline Hispanic/Latin(x) & $18 \%$ & $18 \%$ & $\mathrm{X}^{2}(1)=.01$ & .93 \\
\hline \multicolumn{5}{|l|}{$\operatorname{Sex}(\%)$} \\
\hline Female & $71 \%$ & $69 \%$ & $\mathrm{X}^{2}(1)=.14$ & .71 \\
\hline Male & $29 \%$ & $31 \%$ & & \\
\hline \multicolumn{5}{|l|}{ Self-Report Measures } \\
\hline Hypomanic Personality Scale & $21.02(7.84)$ & $21.34(9.09)$ & $\mathrm{t}(350)=-.35$ & .73 \\
\hline MASQ Anhedonic Depression & $20.03(6.39)$ & $19.52(6.48)$ & $\mathrm{t}(346)=.70$ & .49 \\
\hline NEO-FFI Neuroticism & $39.93(8.59)$ & $39.34(8.37)$ & $\mathrm{t}(350)=.62$ & .54 \\
\hline
\end{tabular}

$* p<.05 \quad * * p<.01 \quad * * * p<.001$ 
Table 2. Direct Effects Between HPS and ESM Items and Indices

\begin{tabular}{lc}
\hline Level 1 Criterion & $\begin{array}{c}\text { Level } 2 \text { Predictor } \\
\gamma_{01}(\mathrm{df}=231)\end{array}$ \\
\hline Affect & HPS \\
High Arousal NA & $.15(.05)^{* *}$ \\
Low Arousal NA & $.06(.05)$ \\
High Arousal PA & $.29(.06)^{* * *}$ \\
Low Arousal PA & $-.03(.06)$ \\
Emotions out of control & $.26\left(.06^{* * *}\right.$ \\
Situation & $.11(.06)$ \\
Current situation is stressful & $-.03(.06)$ \\
Experience pleasant & $.12(.06)^{*}$ \\
Since the last beep, experiences unpleasant & $-.10(.07)$ \\
Since the last beep, experiences pleasant & $-.01(.06)$ \\
Enjoying current activity &
\end{tabular}


Table 3. NA and PA Emotion Reactivity in Daily Life

\begin{tabular}{|c|c|c|c|c|c|c|c|c|c|}
\hline \multirow[b]{3}{*}{ Outcome } & \multicolumn{4}{|c|}{ Fixed Effects } & \multicolumn{4}{|c|}{ Random Effects } & \multirow{3}{*}{$\frac{\text { Effect size }}{\text { ICC }}$} \\
\hline & \multirow[b]{2}{*}{$\gamma$} & \multirow[b]{2}{*}{$\mathrm{B}$} & \multirow[b]{2}{*}{$\mathrm{SE}$} & \multirow[b]{2}{*}{$\mathrm{p}$} & \multicolumn{2}{|c|}{ Intercept } & \multicolumn{2}{|c|}{ Residual } & \\
\hline & & & & & $\hat{\tau}^{2}$ & $\mathrm{SD}$ & $\hat{\sigma}^{2}$ & $\mathrm{SD}$ & \\
\hline \multirow{5}{*}{$\mathrm{NA}_{\mathrm{HA}}$} & Intercept & 2.16 & .06 & $<.001$ & .63 & .79 & 4.53 & 2.13 & .12 \\
\hline & Stress & .28 & .02 & $<.001$ & .03 & .18 & & & \\
\hline & $\mathrm{NA}_{\mathrm{HA}} \_$lag & .00 & .00 & .68 & & & & & \\
\hline & $\mathrm{HPS}$ & .13 & .05 & .02 & & & & & \\
\hline & HPS *Stress & .03 & .02 & .13 & & & & & \\
\hline \multirow[t]{5}{*}{$\mathrm{NA}_{\mathrm{HA}}$} & Intercept & 2.17 & .06 & $<.001$ & .63 & .79 & 4.50 & 2.12 & .12 \\
\hline & Unpleasant Event & .35 & .02 & $<.001$ & .03 & .17 & & & \\
\hline & $\mathrm{NA}_{\mathrm{HAl}}$ lag & .00 & .00 & .53 & & & & & \\
\hline & HPS & .13 & .05 & .02 & & & & & \\
\hline & HPS*Unpleasant Event & .02 & .02 & .34 & & & & & \\
\hline \multirow[t]{5}{*}{$\mathrm{NA}_{\mathrm{LA}}$} & Intercept & 2.62 & .06 & $<.001$ & .67 & .82 & 5.41 & 2.33 & .11 \\
\hline & Stress & .14 & .01 & $<.001$ & .01 & .12 & & & \\
\hline & $\mathrm{NA}_{\text {LA_lag }}$ & .00 & .00 & .83 & & & & & \\
\hline & HPS & .05 & .06 & .43 & & & & & \\
\hline & HPS *Stress & .02 & .01 & .23 & & & & & \\
\hline \multirow[t]{5}{*}{$\mathrm{NA}_{L A}$} & Intercept & 2.62 & .06 & $<.001$ & .67 & .82 & 5.37 & 2.32 & .11 \\
\hline & Unpleasant Event & .28 & .02 & $<.001$ & .01 & .10 & & & \\
\hline & $\mathrm{NA}_{\mathrm{LA}}$ lag & .00 & .00 & .63 & & & & & \\
\hline & HPS & .04 & .06 & .43 & & & & & \\
\hline & HPS*Unpleasant Event & .00 & .02 & .96 & & & & & \\
\hline \multirow{5}{*}{$\begin{array}{l}\text { Emotions } \\
\text { out of } \\
\text { Control }\end{array}$} & Intercept & 2.14 & .06 & $<.001$ & .88 & .94 & 2.02 & 1.42 & .29 \\
\hline & Stress & .20 & .01 & $<.001$ & .04 & .19 & & & \\
\hline & Emotions_lag & .00 & .00 & .05 & & & & & \\
\hline & HPS & .25 & .06 & $<.001$ & & & & & \\
\hline & HPS *Stress & .04 & .01 & .02 & & & & & \\
\hline
\end{tabular}




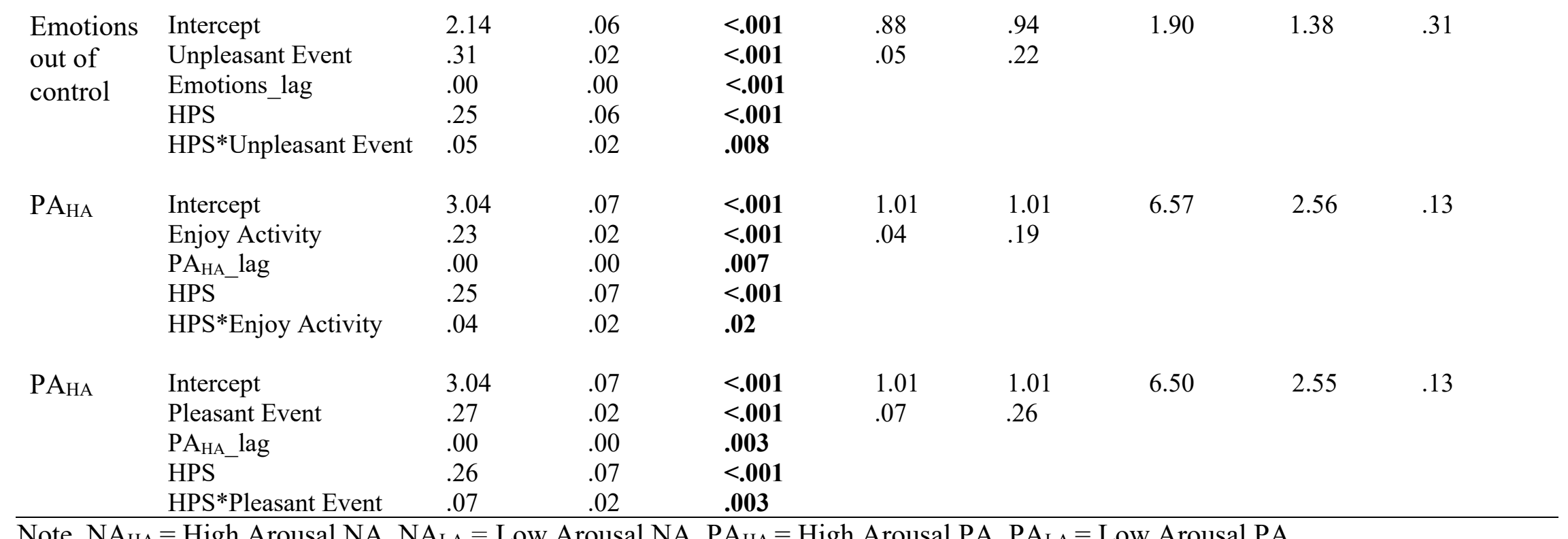

Note. $\mathrm{NA}_{\mathrm{HA}}=$ High Arousal NA, NA $\mathrm{LA}=$ Low Arousal NA, PA $\mathrm{HA}=$ High Arousal PA, PA $\mathrm{LA}=$ Low Arousal PA 
Table 4. Associations of Within-Day Emotion Dynamics and Bipolar Spectrum Psychopathology

\begin{tabular}{|c|c|c|c|c|c|c|c|c|c|}
\hline \multirow[b]{3}{*}{ Outcome } & \multicolumn{4}{|c|}{ Fixed Effects } & \multicolumn{4}{|c|}{ Random Effects } & \multirow{3}{*}{$\frac{\text { Effect Size }}{\text { ICC }}$} \\
\hline & \multirow{2}{*}{\multicolumn{2}{|c|}{$\gamma$}} & \multirow[b]{2}{*}{$\mathrm{SE}$} & \multirow[b]{2}{*}{$\mathrm{p}$} & \multicolumn{2}{|c|}{ Intercept } & \multicolumn{2}{|c|}{ Residual } & \\
\hline & & & & & $\hat{\tau}^{2}$ & SD & $\hat{\sigma}^{2}$ & SD & \\
\hline \multicolumn{10}{|c|}{ Within-Person Variance (WPV) } \\
\hline \multirow[t]{3}{*}{$\mathrm{NA}_{\mathrm{HA}}$} & Intercept & .37 & .05 & $<.001$ & -- & -- & -- & -- & -- \\
\hline & HPS & .04 & .02 & .03 & & & & & \\
\hline & Mean NA & .25 & .02 & $<.001$ & & & & & \\
\hline \multirow[t]{3}{*}{$\mathrm{NA}_{\text {LA }}$} & Intercept & .67 & .06 & $<.001$ & -- & -- & -- & -- & -- \\
\hline & HPS & .04 & .02 & .03 & & & & & \\
\hline & Mean NALA & .12 & .02 & $<.001$ & & & & & \\
\hline \multirow[t]{3}{*}{$\mathrm{PA}_{\mathrm{HA}}$} & Intercept & .73 & .07 & $<.001$ & -- & -- & -- & -- & -- \\
\hline & HPS & .03 & .02 & .26 & & & & & \\
\hline & Mean $\mathrm{PA}_{\mathrm{HA}}$ & .09 & .02 & $<.001$ & & & & & \\
\hline \multirow{3}{*}{$\mathrm{PA}_{\mathrm{LA}}$} & Intercept & .97 & .11 & $<.001$ & -- & -- & -- & -- & -- \\
\hline & HPS & .01 & .02 & .62 & & & & & \\
\hline & Mean PALA & .05 & .02 & .03 & & & & & \\
\hline \multicolumn{10}{|c|}{ Adjusted Squared Successive Differences (ASSD) } \\
\hline \multirow[t]{3}{*}{$\mathrm{NA}_{\mathrm{HA}}$} & Intercept & 1.40 & .06 & $<.001$ & .66 & .81 & 7.64 & 2.76 & .20 \\
\hline & HPS & .18 & .06 & .002 & & & & & \\
\hline & Mean NAHA & .72 & .02 & $<.001$ & & & & & \\
\hline \multirow{3}{*}{$\mathrm{NA}_{\mathrm{LA}}$} & Intercept & 1.60 & .05 & $<.001$ & .50 & .71 & 6.65 & 2.58 & .07 \\
\hline & HPS & .15 & .05 & .004 & & & & & \\
\hline & Mean NALA & .38 & .02 & $<.001$ & & & & & \\
\hline \multirow[t]{3}{*}{$\mathrm{PA}_{\mathrm{HA}}$} & Intercept & 1.41 & .07 & $<.001$ & .91 & .95 & 7.85 & 2.80 & .10 \\
\hline & HPS & .15 & .07 & .02 & & & & & \\
\hline & Mean $\mathrm{PA}_{\mathrm{HA}}$ & .41 & .03 & $<.001$ & & & & & \\
\hline $\mathrm{PA}_{\mathrm{LA}}$ & Intercept & 2.10 & .08 & $<.001$ & 1.39 & 1.18 & 14.20 & 3.77 & .09 \\
\hline
\end{tabular}




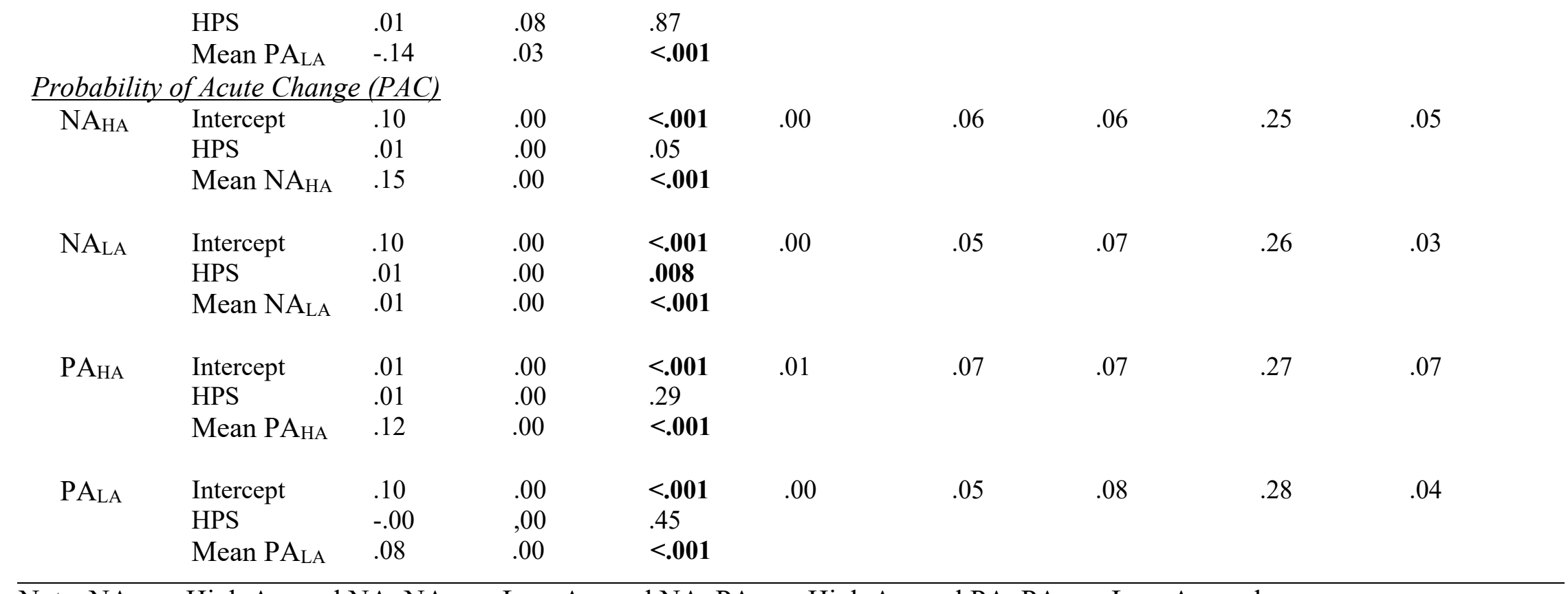

Note. $\mathrm{NA}_{\mathrm{HA}}=$ High Arousal NA, NA $\mathrm{NA}=$ Low Arousal NA, $\mathrm{PA}_{\mathrm{HA}}=$ High Arousal PA, PA $\mathrm{LA}=$ Low Arousal 
Table 5. Associations of Between-Day Emotion Dynamics and Bipolar Spectrum Psychopathology

\begin{tabular}{|c|c|c|c|c|c|c|c|c|c|}
\hline \multirow[b]{3}{*}{ Outcome } & \multicolumn{4}{|c|}{ Fixed Effects } & \multicolumn{4}{|c|}{ Random Effects } & \multirow{3}{*}{$\frac{\text { Effect Size }}{\text { ICC }}$} \\
\hline & \multirow[b]{2}{*}{$\gamma$} & \multirow[b]{2}{*}{$\mathrm{B}$} & \multirow[b]{2}{*}{ SE } & \multirow[b]{2}{*}{$\mathrm{p}$} & \multicolumn{2}{|c|}{ Intercept } & \multicolumn{2}{|c|}{ Residual } & \\
\hline & & & & & $\hat{\tau}^{2}$ & $\mathrm{SD}$ & $\hat{\sigma}^{2}$ & SD & \\
\hline \multicolumn{10}{|c|}{ Within-Person Variance (WPV) } \\
\hline \multirow[t]{3}{*}{$\mathrm{NA}_{\mathrm{HA}}$} & Intercept & .69 & .02 & $<.001$ & .08 & .28 & .14 & .37 & .36 \\
\hline & HPS & .06 & .02 & .002 & & & & & \\
\hline & Mean NA & .30 & .01 & $<.001$ & & & & & \\
\hline \multirow[t]{3}{*}{$\mathrm{NA}_{\mathrm{LA}}$} & Intercept & .79 & .02 & $<.001$ & .06 & .24 & .13 & .36 & .31 \\
\hline & HPS & .04 & .02 & .007 & & & & & \\
\hline & Mean NA LA & .15 & .00 & $<.001$ & & & & & \\
\hline \multirow[t]{3}{*}{$\mathrm{PA}_{\mathrm{HA}}$} & Intercept & .78 & .02 & $<.001$ & .08 & .29 & .14 & .38 & .37 \\
\hline & HPS & .05 & .02 & .008 & & & & & \\
\hline & Mean $\mathrm{PA}_{\mathrm{HA}}$ & .12 & .00 & $<.001$ & & & & & \\
\hline \multirow[t]{3}{*}{$\mathrm{PA}_{\mathrm{LA}}$} & Intercept & .95 & .02 & $<.001$ & .09 & .30 & .20 & .45 & .32 \\
\hline & HPS & .01 & .02 & .76 & & & & & \\
\hline & Mean PALA & -.08 & .00 & $<.001$ & & & & & \\
\hline \multicolumn{10}{|c|}{ Adjusted Squared Successive Differences (ASSD) } \\
\hline \multirow[t]{3}{*}{$\mathrm{NA}_{\mathrm{HA}}$} & Intercept & 1.26 & .06 & $<.001$ & .75 & .86 & 2.37 & 1.54 & .24 \\
\hline & HPS & .18 & .06 & .003 & & & & & \\
\hline & Mean NA & .83 & .02 & $<.001$ & & & & & \\
\hline \multirow[t]{3}{*}{$\mathrm{NA}_{\mathrm{LA}}$} & Intercept & 1.42 & .05 & $<.001$ & .59 & .77 & 1.95 & 1.39 & .23 \\
\hline & HPS & .15 & .05 & .003 & & & & & \\
\hline & Mean NALA & .44 & .02 & $<.001$ & & & & & \\
\hline \multirow[t]{3}{*}{$\mathrm{PA}_{\mathrm{HA}}$} & Intercept & 1.45 & .07 & $<.001$ & .98 & .99 & 2.42 & 1.56 & .29 \\
\hline & HPS & .17 & .07 & .01 & & & & & \\
\hline & Mean $\mathrm{PA}_{\mathrm{HA}}$ & .35 & .02 & $<.001$ & & & & & \\
\hline PA $_{\text {LA }}$ & Intercept & 2.10 & .08 & $<.001$ & 1.58 & 1.26 & 4.59 & 2.14 & .26 \\
\hline
\end{tabular}




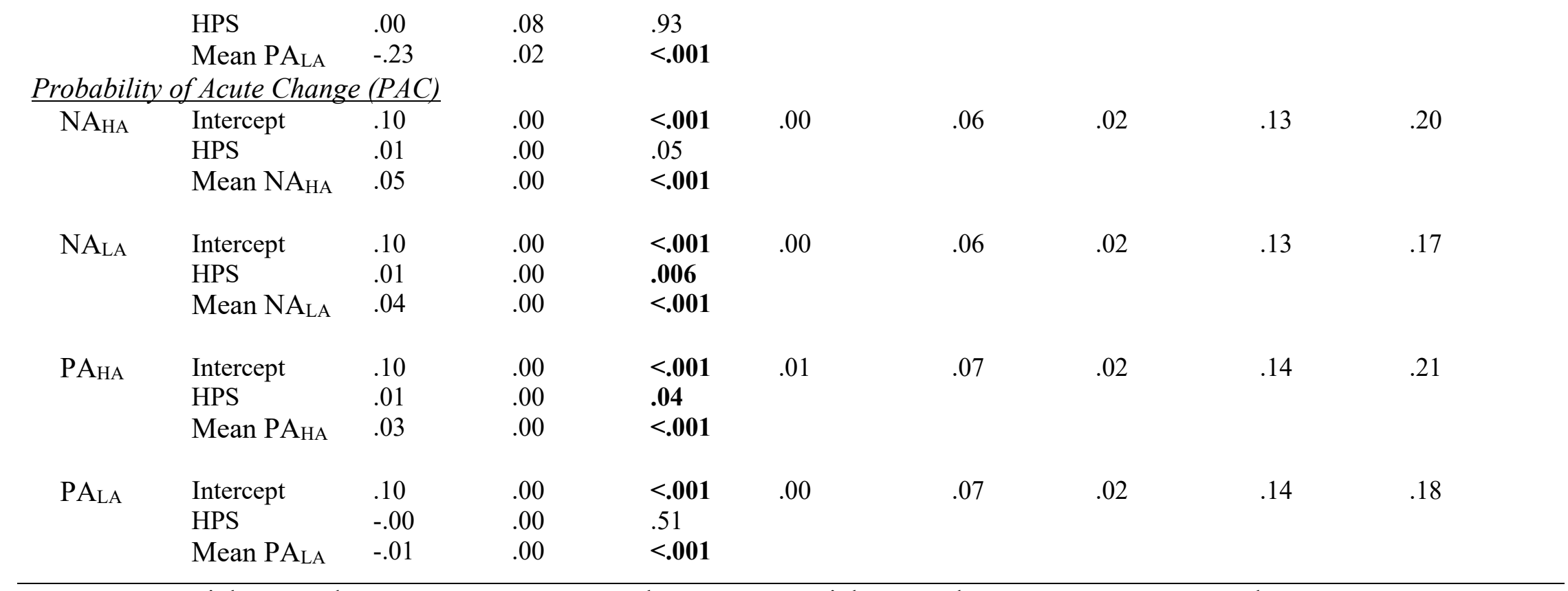

Note. $\mathrm{NA}_{\mathrm{HA}}=$ High Arousal NA, NA $\mathrm{NA}=$ Low Arousal NA, $\mathrm{PA}_{\mathrm{HA}}=$ High Arousal PA, PA $\mathrm{LA}=$ Low Arousal 

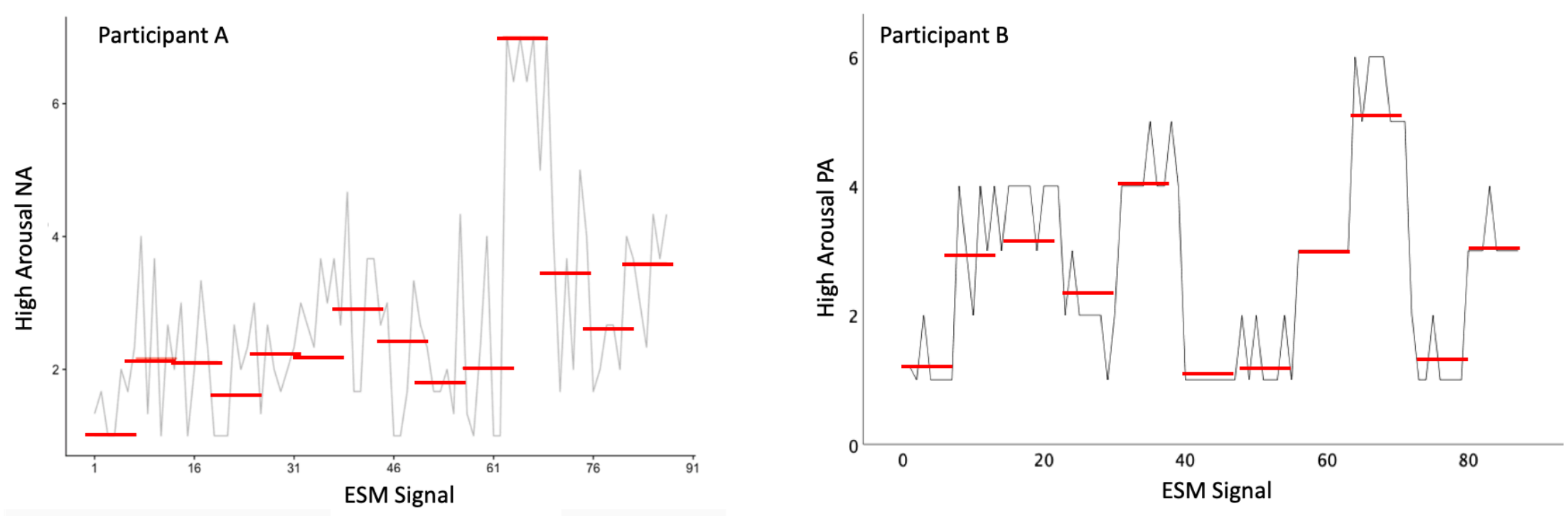

Figure 1. Within vs. between-day instability of NA and PA in two participants.

These figures show two different participants who had patterns of dynamics that reflect differential within vs. between-day instability as measured by PAC in NA (Participant A) and PA (Participant B). Participant A shows large increases in NA from moment to moment (within day) and large increases in NA from one day to the next (between day). Participant B did not show significant large increases in PA from moment to moment (within day) but did show large increases in PA from one day to the next (between day). Horizontal lines represent mean NA and PA for that day whereas the timeseries represents each individual report of NA or PA from moment-to-moment. 

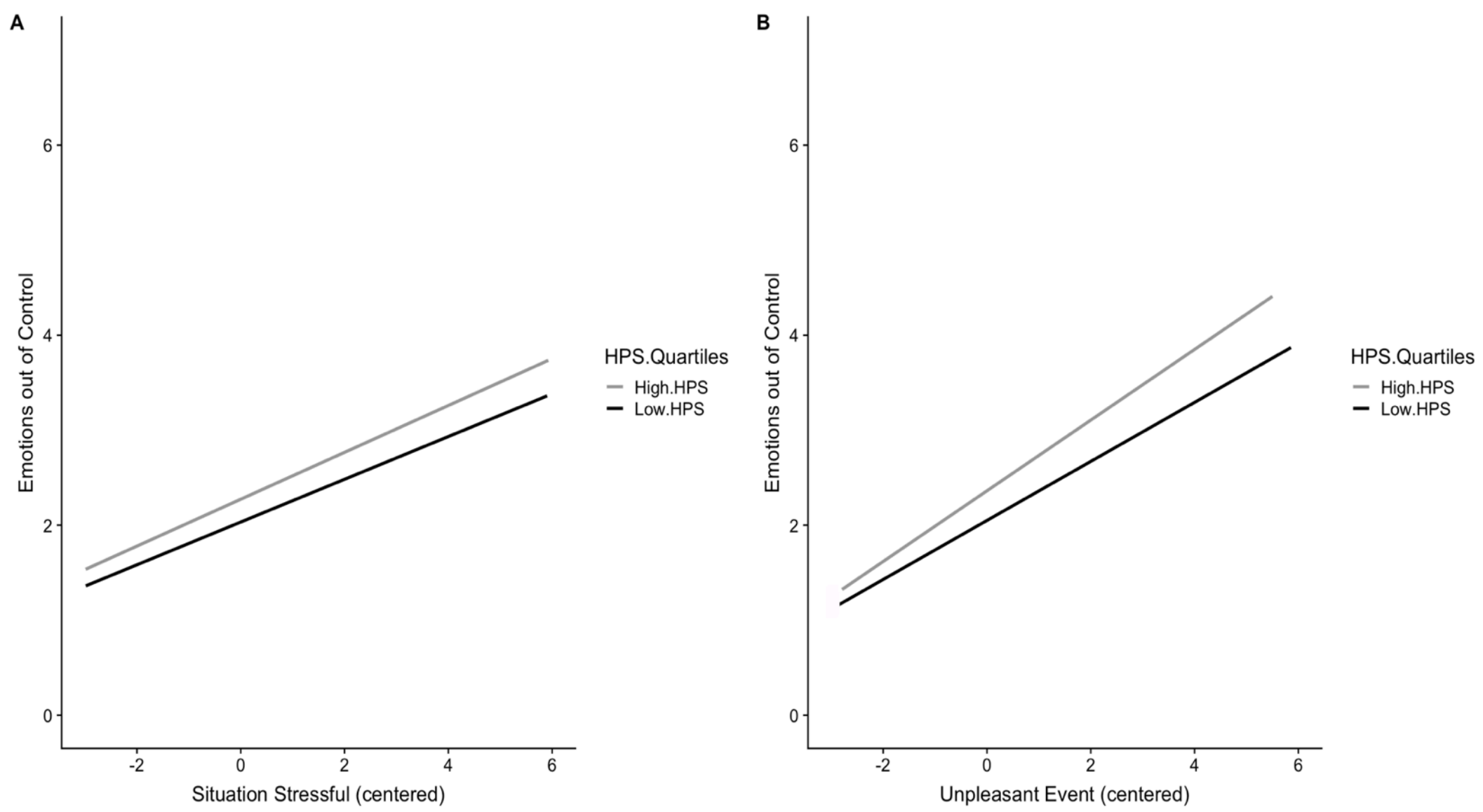

Figure 2. Negative Affect Reactivity in Daily Life

All analyses examined HPS scores continuously. In order to visually display interactions, HPS scores we present the association of the level 1 predictor and criterion at the highest and lowest quartile of HPS scores. 
A

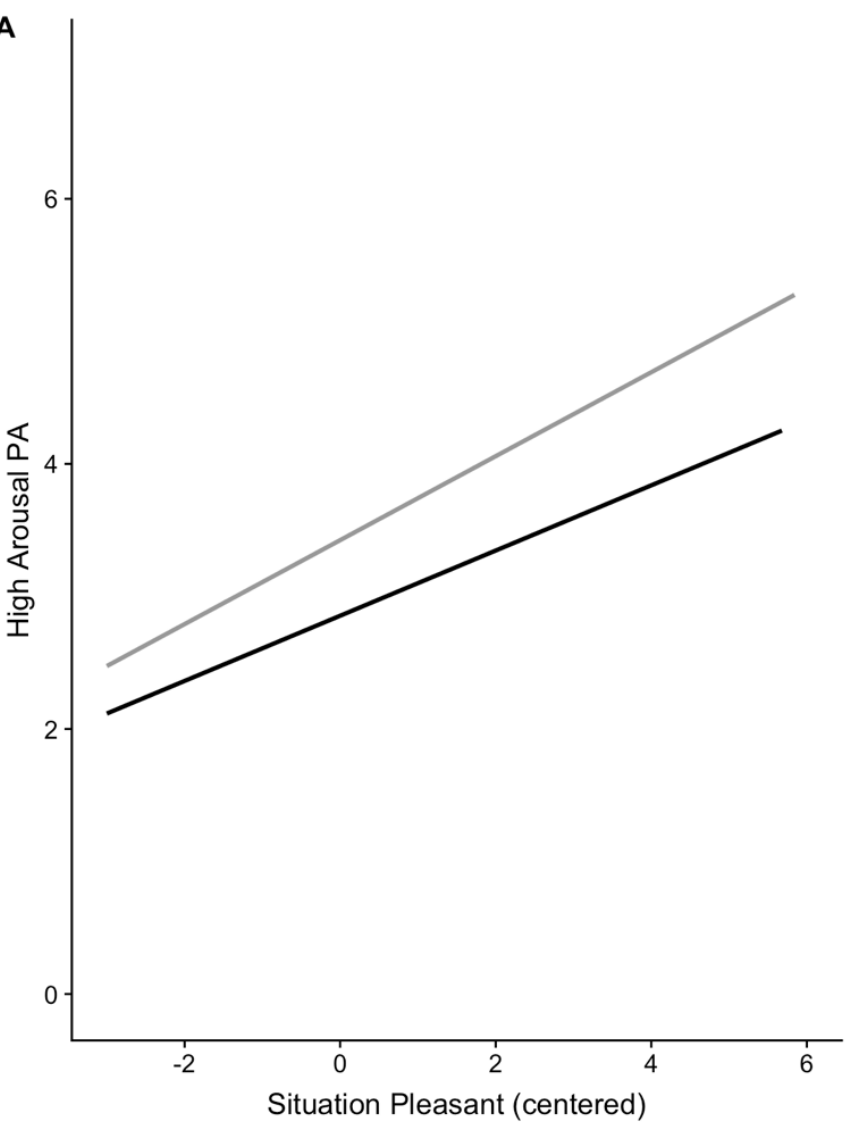

B

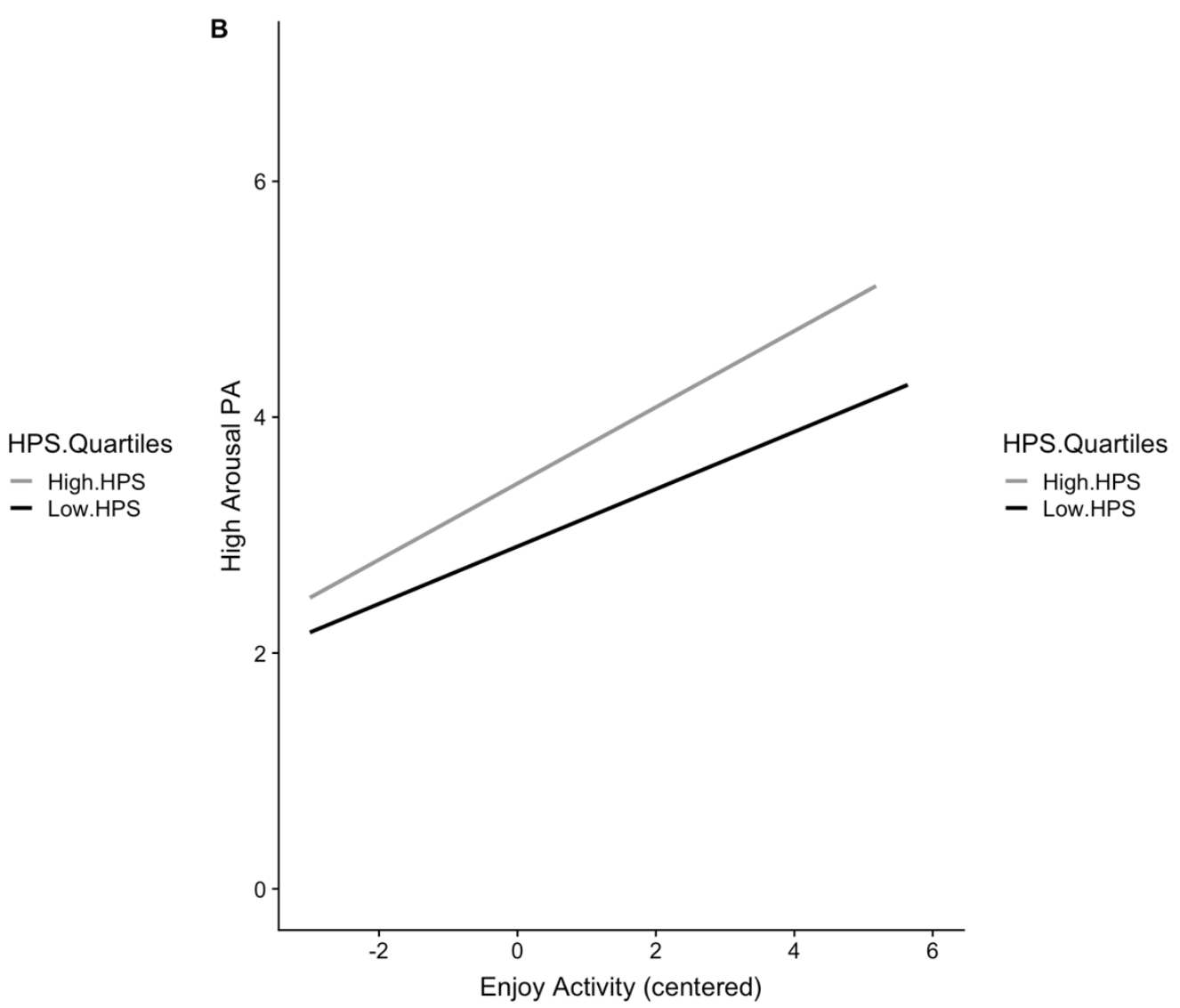

Figure 3. Positive Affect Reactivity in Daily Life

Note. All analyses examined HPS scores continuously. In order to visually display interactions, HPS scores we present the association of the level 1 predictor and criterion at the highest and lowest quartile of HPS scores. 